\title{
ODONTOPLASTIA E SEU EFEITO NA NUTRIÇÃO DE CAVALOS
}

\section{Odontoplasty and its effect on horse nutrition}

Luiz Antonio Jorge de Moraes Filho ${ }^{1}$, $K a ́ t i a ~ F e l t r e{ }^{1}$, Lizzie de Oliveira Dietrich², Camila Bianconi ${ }^{1}$, Alexandre Augusto de Oliveira Gobesso ${ }^{1}$

$$
{ }^{1} \text { Faculdade de Medicina Veterinária e Zootecnia (USP), }{ }^{2} \text { Equident Odontologia Equina, }
$$

*Autor para correspondência: katiafeltre@yahoo.com.br

RESUMO: O objetivo do trabalho foi investigar as implicações de um tratamento odontológico (odontoplastia) em cavalos adultos (80 \pm 7 meses) sobre os parâmetros digestivos, metabólicos e comportamentais. Foram utilizados oito cavalos, machos, castrados, da raça Puro Sangue Árabe, nunca submetidos a tratamento odontológico. A proporção (\%/\%) concentrado/volumoso foi de 37,5/62,5. Para a avaliação dos parâmetros fecais, sanguíneos e de digestibilidade, os cavalos foram divididos em D0 (grupo controle; sem odontoplastia), D20 (20 dias após a odontoplastia) e D40 (40 dias após a odontoplastia). Para o comportamento alimentar, os animais foram divididos em grupo controle (sem odontoplastia), T1 (imediatamente após à odontoplastia) e T2 (20 dias após a odontoplastia). Foram realizados três períodos experimentais (15 dias para adaptação à dieta e 5 dias para coleta de sangue e coleta total de fezes). Foi utilizado o delineamento inteiramente ao acaso e as médias foram comparadas pelo teste de Tukey com $P<0,05$. A odontoplastia removeu ganchos, ondas, cristas transversas e degraus com a redução de pontas excessivas de esmalte e ajuste da oclusão. Os animais submetidos à odontoplastia apresentaram maior consumo de água (em L/dia) em T1 e T2, menor coeficiente de digestibilidade aparente (\%) da fibra em detergente ácido em D20 e D40, menor concentração fecal (mmol) de lactato (D20 e D40) e maior de propionato (D0 e D20). Os resultados sugerem que a odontoplastia, apesar de reduzir o coeficiente de digestibilidade aparente da fibra em detergente ácido, melhorou as condições fermentativas no intestino grosso sem alterar o $\mathrm{pH}$ e causar distúrbios gastrintestinais.

Palavras-chave: alimentação; cavalos; comportamento alimentar; digestibilidade; tratamento dentário.

ABSTRACT: The aim of this study was to investigate the implications of a dental treatment (odontoplasty) in adult horses on the digestive, metabolic and behavioral parameters. We used eight gelding male purebred Arabian horses (80 \pm 7 month), never undergoing dental treatment. The concentrate / roughage ratio $(\% / \%)$ was 37.5/62.5. For fecal, blood and digestibility parameters evaluation, horses were divided in D0 (control group; without odontoplasty), D20 (20 days after odontoplasty) and D40 (40 days after odontoplasty). For the feeding behavior, they were divided into control group (without odontoplasty), T1 (immediately after odontoplasty) and T2 (20 days after odontoplasty). We performed three experimental periods with 20 days each (15 days for diet adaptation and 5 days for blood and fecal collections). A completely randomized design was used, and the means were compared by the Tukey test with $P<0.05$. The odontoplasty removed hooks, waves, transverse ridges, stairs with reduction of excessive enamel tips and adjustment of the occlusion. Animals submitted to odontoplasty presented higher water consumption (in L/day) in 
T1 and T2, the lowest coefficient of apparent digestibility (\%) of acid detergent fiber in D20 and D40, the lower fecal concentration (mmol) of lactate (D20 and D40) and the higher propionate concentration (D0 and D20). The results suggest that odontoplasty, although reducing the apparent digestibility coefficient of the acid detergent fiber, improved the fermentative conditions in the large intestine without alterations of $\mathrm{pH}$ and gastrointestinal disorders.

Keywords: dental treatment; digestibility; equine; feed; feeding behavior. 


\section{INTRODUÇÃO}

A maior concentração de cavalos no setor pecuário, bem como sua utilização no esporte e no lazer, exige maior empenho dos proprietários e veterinários para maximizar o desempenho e reduzir ou amenizar as enfermidades que acometem esses animais. A domesticação e estabulação restringiram o tamanho das áreas e o tempo destinado ao pastejo, além de predeterminar os horários, quantidade, qualidade e o tipo do alimento oferecido (Dittrich et al., 2010). Essa condição de manejo ocasionou mudanças nos hábitos e nos padrões digestivos, incluindo-se nessa última o surgimento de desequilíbrios oclusais.

A condição dentária influencia diretamente na digestão e, consequentemente, na saúde intestinal, uma vez que uma mastigação eficiente resulta em melhor trituração dos alimentos com redução no tamanho de partículas e aumento na digestibilidade dos nutrientes (Araújo et al., 2018; Di Filippo et al., 2018). Além disso, é de extrema importância entender a ação e propósitos dos freios, embocaduras e acessórios, não apenas para fornecer cuidados de saúde ideais para a boca dos cavalos, mas também para fornecer o tipo de tratamento dentário necessário para ajudar os cavalos a se comportarem da maneira mais confortável e com o melhor desempenho possível, pois qualquer alteração na cavidade oral pode resultar em perda de peso devido à má alimentação, acúmulo de alimento na boca e alterações comportamentais como reação à embocadura e problemas considerados de temperamento ou doma (Bennett, 2005). A odontoplastia é considerada uma técnica que contribui para saúde do cavalo. Baseia-se em retomar o equilíbrio da oclusão (mordida), pelo desgaste da coroa clínica do dente. Para isto, devem ser feitas correções das anormalidades dentárias de molares e incisivos com o objetivo de melhorar a qualidade de vida dos cavalos, proporcionando saúde, bem-estar e, consequentemente, melhor desempenho (Dietrich, 2003).

Assim, o objetivo da pesquisa foi fornecer informações sobre os parâmetros fecais, sanguíneos, comportamentais e de digestibilidade dos nutrientes de cavalos adultos submetidos ao procedimento de equilíbrio oclusal (Odontoplastia).

\section{MATERIAL E MÉTODOS}

A pesquisa foi realizada na Faculdade de Medicina Veterinária e Zootecnia (USP), Pirassununga, SP

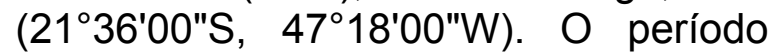
experimental foi de julho a outubro de 2015. O Comitê de Ética no Uso de Animais (CEUA), aprovou a realização do trabalho (protocolo CEUA/FMVZ $\mathrm{n}^{\circ}$. 9760180815).

Foram utilizados oito cavalos, machos, castrados, da raça Puro Sangue Árabe (idade: $80 \pm 7$ meses; peso corporal: $460 \pm 28 \mathrm{~kg}$ ). Os cavalos apresentavam alterações moderadas na superfície oclusal de molares e incisivos, porém, nenhum deles havia recebido tratamento dentário previamente. Os animais foram mantidos em baias individuais de aproximadamente $9 \mathrm{~m}^{2}$ com piso de concreto coberto com cama de maravalha e cocho de água com volume conhecido. Foram vermifugados com produto a base de Ivermectina, pulverizados com carrapaticida a base de Cipermetrina e vacinados contra tétato, influenza e encefalomielite previamente ao experimento. A higidez foi avaliada por meio de exame clínico (tempo de preenchimento capilar, frequência cardíaca e respiratória, temperatura e hidratação) e hematológico (série vermelha e branca, linfócitos, monócitos, granulócitos, 
hematócrito, plaquetas, etc.) no início e ao final de cada fase. Todos os animais permaneceram clinicamente saudáveis e mantiveram-se hígidos durante a realização do estudo.

$$
\text { A proporção }
$$

concentrado/volumoso foi de $37,5 / 62,5$. O consumo de matéria seca foi $2 \%$ do PC, de acordo com o Nutrient Requirements of Horses (NRC, 2007) para cavalos adultos em manutenção. As dietas foram fornecidas duas vezes ao dia (07h00min e 19h00min). Água e suplemento mineral foram fornecidos ad libitum. A composição bromatológica dos alimentos pode ser observada na Tabela 1.

Tabela 1 - Composição nutricional da dieta utilizada no período experimental.

\begin{tabular}{lccc}
\hline \multicolumn{1}{c}{ Nutriente (\%) } & Feno $^{\mathbf{1}}$ & Concentrado $^{2}$ & Dieta Total \\
\hline Matéria seca & 92,62 & 88,51 & 91,08 \\
Proteína bruta & 12,59 & 14,73 & 13,39 \\
Fibra em detergente neutro & 76,41 & 18,25 & 54,60 \\
Fibra em detergente ácido & 34,77 & 4,83 & 23,54 \\
Extrato etéreo & 0,91 & 6,20 & 2,89 \\
\hline
\end{tabular}

${ }^{1}$ Cynodon dactylon cv. Tifton 85; ${ }^{2}$ Comercial peletizado

Foram realizados três períodos com 20 dias cada (15 para adaptação à dieta e 5 para coleta de sangue e fezes). Os parâmetros sanguíneos, fecais e de digestibilidade aparente foram avaliados no D0, D20 e D40. A análise do comportamento alimentar foi realizada nos momentos T1 e T2. Na figura 1, é possível observar um esquema dos períodos experimentais e das coletas de amostras.

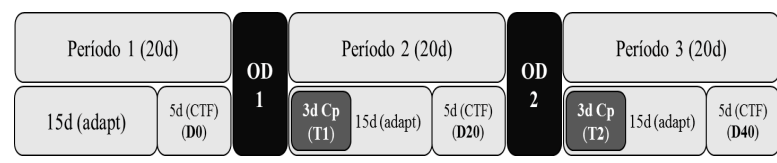

Figura 1 - Esquema dos períodos experimentais e coletas de amostras realizados em cavalos submetidos ou não à odontoplastia.

Legenda: Adapt = adaptação. OD = odontoplastia. $\mathrm{d}=$ dias. $\mathrm{CTF}=$ coleta total de fezes. $\mathrm{Cp}=$ análise do comportamento alimentar.
As frequências (\%) dos eventos comportamentais foi mensurada nos três dias subsequentes à odontoplastia, minuto a minuto, das $07 \mathrm{~h} 00 \mathrm{~min}$ às $11 \mathrm{~h} 00 \mathrm{~min}$ e das $19 \mathrm{~h} 00 \mathrm{~min}$ às $23 \mathrm{~h} 00 \mathrm{~min}$. Os animais foram divididos em: grupo controle (GC); grupo tratado 1 (T1); e grupo tratado 2 (T2). Os eventos comportamentais foram classificados como: comendo feno (CF), comendo concentrado (CC), lambendo sal (LS), ingerindo água (IA), comendo na porta e observando o exterior (CP), mordendo a porta (MP) e repouso (ócio). A quantidade de água ingerida, em L/dia, foi contabilizada por diferença a cada 12 horas.

Para a digestibilidade aparente dos nutrientes, a cama de maravalha foi removida e as fezes foram coletadas do chão e acondicionadas em sacos plásticos. A cada 24 horas as amostras foram pesadas, homogeneizadas e uma alíquota de $10 \%$ foi retirada e congelada a $-20^{\circ} \mathrm{C}$. Ao final do período experimental, as amostras dos três períodos foram descongeladas, homogeneizadas e uma alíquota de 500 $g$ foi seca em estufa de circulação forçada de ar a $55^{\circ} \mathrm{C}$ até peso constante. Foram calculados os teores de matéria seca (MS), matéria orgânica (MO), proteína bruta (PB), matéria mineral ( $\mathrm{MM})$ e extrato etéreo ( $E E)$ de acordo com AOAC (2005). As análises de fibra em detergente neutro (FDN) e ácido (FDA) foram determinadas pelo método de partição de fibras (Van Soest et al., 1991). Os coeficientes de digestibilidade aparente (CDA) dos nutrientes foram obtidos por meio da equação: CDA $(\%)=[\mathrm{NI}-\mathrm{NE} / \mathrm{NI}] \mathrm{x}$ 100 , onde $\mathrm{NI}$ é a quantidade $(\mathrm{g})$ de nutriente ingerido (descontando-se as sobras) e NE, a quantidade (g) de nutriente excretado nas fezes.

As amostras sanguíneas foram coletadas por venopunção da veia jugular, acondicionadas em tubos contendo fluoreto de sódio e EDTA 
(plasma) ou tubos sem anticoagulantes (soro) e mantidas em repouso por aproximadamente 20 minutos. Em seguida, foram centrifugadas por 10 minutos a $1.800 \times \mathrm{g}$. O sobrenadante foi transferido para microtubos de $1,5 \mathrm{~mL}$ e congelado $\mathrm{a}-20^{\circ} \mathrm{C}$.

A área total abaixo da curva (AAC) de glicose (plasma) e insulina (soro) foi calculada a partir do sangue coletado em cinco momentos: às 6h30min (30 minutos antes do fornecimento da dieta), 07h30min, 08h30min, 09h30min e 10h30min. A concentração de glicose foi determinada pelo método enzimático (kit comercial Labtest) e a insulina, por quimiluminescência (kit comercial Access Ultrasensitive Insulin; Beckman Coulter, Inc.). A AAC de glicose e insulina foi calculada pela área do trapézio proposto por Matthews et al. (1990).

As concentrações de colesterol total (CT), Lipoproteínas (Lipoproteína de alta densidade - HDL; Lipoproteína de baixa densidade - LDL; e Lipoproteína de muito baixa densidade VLDL) e de Triglicérides (TG) no soro foram colhidas 30 minutos antes do trato da manhã (6h30min), como sugerido por Marchello et al. (2000). O CT e o TG foram determinados pelo método Colorimétrico (Enzimático de Trinder, kits: Colesterol Liquiform e Triglicérides Liquiform; Labtest Diagnóstica). A HDL foi quantificada pelo método Colorimétrico (Acelerador - Detergente Seletivo, kit: HDL LE; Labtest Diagnóstica). O valor de LDL foi calculado seguindo a equação de Friedewald (LDL = CT - HDL - TG/5), onde $\mathrm{TG} / 5$ representa o colesterol ligado à VLDL.

Foram coletados $10 \mathrm{~g}$ e $2 \mathrm{~g}$ de fezes, respectivamente, para a análise de ácidos graxos de cadeia curta (AGCC; acético, propiônico e butírico) e lactato. Para os AGCC, as fezes foram diluídas em $20 \mathrm{~mL}$ de água destilada, homogeneizadas, coadas e, $4 \mathrm{~mL}$ de amostra foram transferidos para tubos sem anticoagulante contendo previamente, $1 \mathrm{~mL}$ de ácido fórmico $\mathrm{PA}$ grau HPLC 98-100\%. Em seguida, os tubos foram centrifugados por 12 minutos a $1.800 \times g$ e $2 \mathrm{~mL}$ do sobrenadante $(2,0 \mathrm{~mL})$ foram transferidos para microtubos $\mathrm{e}$ congelados a $-20^{\circ} \mathrm{C}$. As concentrações de AGCC foram medidas por cromatografia em fase gasosa (GC2014, Shimadzu, Japão) por meio de coluna capilar a $145^{\circ} \mathrm{C}$ (isotérmica) e um injetor split/splitless e detector dual FID a $250^{\circ} \mathrm{C}$, utilizando o método descrito por Erwin et al. (1961), adaptado por Getachew et al. (2002). O software GCSolution ${ }^{\circledR}$ (Shimadzu, Japão) foi utilizado para os cálculos. O lactato foi determinado por espectrofotometria para fluidos biológicos, proposto por Pryce (1969).

$\mathrm{O} \mathrm{pH}$ fecal foi aferido por 12 horas (7:00 às 19:00). As amostras (50 g) foram coletadas 1 a 2 minutos após a defecação, tomando-se o cuidado de retirar aquelas do centro da pilha que não tinham entrado em contato com o piso da baia ou que continham material estranho. Em seguida, foram diluídas em $50 \mathrm{~mL}$ de água destilada, homogeneizadas e, logo após o eletrodo do pHmetro (Quimis Q400AS) foi imergido para leitura do $\mathrm{pH}$ (Hydock et al., 2014). Os valores foram enquadrados em quatro faixas de horário de três horas (F1: 7h-10h; F2: 10h-13h; F3:13h-16h; F4: 16h-19h).

A odontoplastia foi realizada em quatro cavalos no OD1 e, em quatro, no OD2 (figura 1). Os cavalos foram alocados em tronco de contenção, e sedados com cloridrato de detomidina na dose de $20 \mu \mathrm{g} / \mathrm{kg}$, de acordo com protocolo de sedação. Após cinco minutos, a cabeça do cavalo foi colocada no apoiador de cabeça. No exame clínico oral, foram avaliados os movimentos de excursão lateral da mandíbula e rostrocaudal, utilizando um 
paquímetro. Com aplicação do espéculo oral e uso de fotóforo, foi realizada a inspeção da cavidade oral, complementada com o uso de um espelho.

Para a odontoplastia foram utilizados os seguintes equipamentos: tronco de contenção para cavalos, cabresto odontológico, paquímetro com escalas em polegadas (até 9) e em milímetros (até 150), espelho e exploradores odontológico para cavalos (Ortovet), fotóforo de cabeça para exploração da cavidade oral, espéculo bucal Mcpherson e canetas odontológicas elétricas com brocas diamantadas e movidas por um motor do tipo retífica Markita, adaptado com chicote de 1,40 $\mathrm{m}$ e pedal de aceleração da marca Foredom. Os modelos utilizados foram: caneta Foredom 44T com disco de corte diamantado para incisivos, caneta odontológica reta de 20 polegadas com broca cilíndrica diamantada, caneta odontológica angulada de 18 polegadas com disco diamantado para desgaste de esmalte dentário, caneta angulada de 18 polegadas com broca vestibular e grosas manuais com lâminas de tungstênio com diferentes angulações para permitir acesso em toda a cavidade oral.

O delineamento experimental adotado foi inteiramente casualizado. Os resultados foram submetidos à análise de variância pelo procedimento PROC MIXED do Statistical Analysis System (versão 9.2), considerando o efeito do tratamento dentário. Para os resultados significativos foi aplicado teste de Tukey utilizando $\mathrm{P}<0,05$ como referência.

\section{RESULTADOS}

\begin{tabular}{lcr}
\multicolumn{1}{c}{ Não } & foram & \multicolumn{2}{c}{ observadas } \\
claudicações, & \multicolumn{2}{c}{ alterações na } \\
consistência & e aspecto das fezes ou \\
problemas & \multicolumn{2}{c}{ fisio-metabólicos }
\end{tabular}

relacionados à dieta, ao manejo ou aos procedimentos utilizados no presente estudo. A odontoplastia removeu saliências oclusais como ganchos, ondas, cristas transversas excessivas, degraus e reduziu pontas excessivas de esmalte em dentes molares. Ajustou a oclusão de molares e incisivos, removeu qualquer contato prematuro entre os dentes e equilibrou os movimentos de excursão (figuras 2 e 3 ).

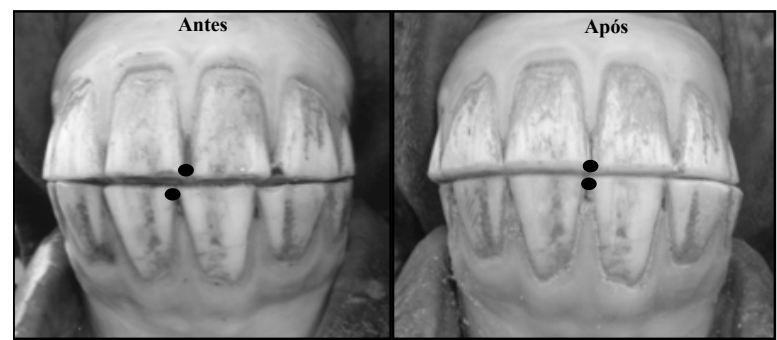

Figura 2 - Ajuste da oclusão antes e após a odontoplastia em cavalos.

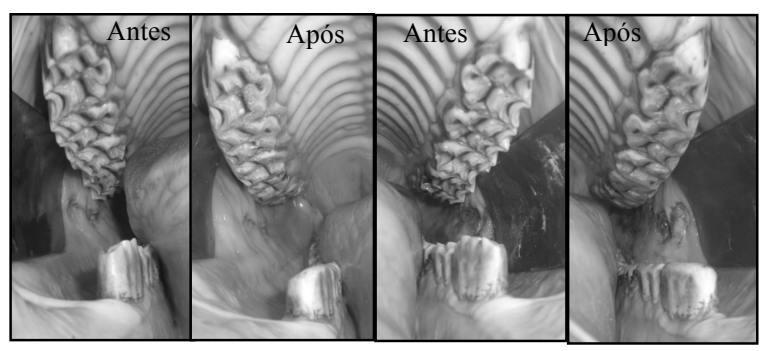

Figura 3 - Redução de ganchos, ondas, cristas transversas, degraus e redução de pontas excessivas de esmalte, antes e após a odontoplastia em cavalos

A odontoplastia não interferiu $(P>0,05)$ nas frequências dos eventos comportamentais dos cavalos. Porém, o consumo de água, em L/dia, foi maior $(p>0,05)$ após a odontoplastia. Foi possível observar discreto aumento e diminuição nas frequências, respectivamente, de consumo do concentrado e de morder a porta em T1 e T2. Os resultados estão apresentados na tabela 2.

Os coeficientes de digestibilidade aparente (CDA) da MS, PB, EE, FDN e $M O$ não foram alterados $(P>0,05)$ pela odontoplastia. Porém, o CDA da FDA foi menor $(P<0,05)$ nos cavalos submetidos 
ao procedimento odontológico (tabela 3).

Tabela 2 - Frequências dos eventos comportamentais e consumo de água de cavalos submetidos ou não à odontoplastia.

\begin{tabular}{lcccccc}
\hline \multicolumn{1}{c}{ Eventos } & $\mathbf{G C}^{1}$ & $\mathrm{T1}^{\mathbf{2}}$ & $\mathrm{T2}^{\mathbf{3}}$ & Média & $\mathrm{EPM}^{4}$ & Valor \\
\hline Ingerindo feno (\%) & 33,52 & 31,74 & 42,39 & 34,85 & 15,33 & 0,56 \\
Ingerindo concentrado (\%) & 7,37 & 10,23 & 10,06 & 9,47 & 2,25 & 0,13 \\
Lambendo sal (\%) Comendo na porta ${ }^{\circ}(\%)$ & 0,55 & 0,41 & 0,30 & 0,42 & 0,33 & 0,61 \\
Mordendo a porta (\%) & 4,97 & 5,80 & 4,92 & 5,37 & 3,16 & 0,88 \\
Ocio (\%) & 0,11 & 0,08 & 0,04 & 0,08 & 0,10 & 0,65 \\
Ingerindo água (\%) & 51,74 & 51,72 & 44,10 & 49,82 & 13,95 & 0,68 \\
Consumo de água (L/dia) & 1,73 & 1,32 & 1,07 & 1,36 & 0,76 & 0,51 \\
\hline
\end{tabular}

${ }^{1}$ Grupo Controle (sem odontoplastia). ${ }^{2}$ Imediatamente após a odontoplastia. ${ }^{32} 20$ dias após a odontoplastia. ${ }^{4}$ Erro Padrão da Média. ${ }^{5}$ Observando o exterior. Médias seguidas por letras minúsculas na linha diferem entre si pelo teste de Tukey $(P<0,05)$.

Os coeficientes de digestibilidade aparente (CDA) da MS, PB, EE, FDN e MO não foram alterados $(P>0,05)$ pela odontoplastia. Porém, o CDA da FDA foi menor $(P<0,05)$ nos cavalos submetidos ao procedimento odontológico (tabela $3)$.

Tabela 3 - Coeficientes de digestibilidade aparente (\%) dos nutrientes da dieta de cavalos submetidos ou não à odontoplastia (média \pm EP).

\begin{tabular}{lcccc}
\hline \multicolumn{1}{c}{ Nutrientes } & $\mathrm{D}^{1}$ & $\mathrm{D}^{1} 0^{2}$ & $\mathrm{D}^{2} 0^{3}$ & Valor de $\mathrm{P}$ \\
\hline Matéria seca & $64,21 \pm 1,00$ & $64,38 \pm 1,23$ & $67,56 \pm 1,73$ & 0,27 \\
Proteína bruta & $71,88 \pm 0,91$ & $73,15 \pm 1,12$ & $74,41 \pm 1,58$ & 0,38 \\
Extrato Etéreo & $83,96 \pm 0,75$ & $82,51 \pm 0,92$ & $83,74 \pm 1,30$ & 0,48 \\
Fibra em detergente neutro & $53,91 \pm 1,45$ & $51,56 \pm 1,78$ & $56,54 \pm 2,51$ & 0,30 \\
Fibra em detergente ácido & $49,80 \pm 1,82_{\mathrm{a}}$ & $40,25 \pm 2,23_{\mathrm{b}}$ & $37,02 \pm 3,16_{\mathrm{b}}$ & $<0,01$ \\
Matéria orgânica & $66,31 \pm 1,00$ & $66,80 \pm 1,23$ & $70,00 \pm 1,74$ & 0,23 \\
\hline
\end{tabular}

${ }^{1}$ Animais sem odontoplastia. ${ }^{2} 20$ dias após a odontoplastia. ${ }^{3} 40$ dias após a odontoplastia. Médias seguidas por letras minúsculas na linha diferem entre si pelo teste de Tukey $(P<0,05)$.

A odontoplastia não interferiu $(P>0,05)$ nos resultados de área abaixo da curva (AAC) de glicose e insulina e nas concentrações de colesterol total (CT), lipoproteína de alta densidade (HDL), lipoproteína de baixa densidade (LDL), lipoproteína de muito baixa densidade (VLDL) e de Triglicérides (TG) dos cavalos. Os 29 resultados podem ser observados na tabela 4.
As concentrações de ácido acético e butírico não foram alterados $(P>0,05)$ pelo procedimento odontológico e não foram observadas diferenças nos valores $\mathrm{pH}$ fecal de acordo com as faixas de horário sugeridas. Porém, a concentração de ácido propiônico foi maior $(P<0,05)$ quarenta dias após a odontoplastia e a concentração de lactato nas fezes apresentou redução $(P<0,05)$ aos 20 e 40 dias após 0 tratamento dentário (tabela 5).

Tabela 4 - Área abaixo da curva de glicose e insulina e concentrações de Triglicérides, colesterol total e lipoproteínas de cavalos submetidos ou não à odontoplastia.

\begin{tabular}{lccccc}
\hline \multicolumn{1}{c}{ Variável } & D0 $^{1}$ & $\mathbf{D 2 0}^{\mathbf{2}}$ & $\mathbf{D 4 0}^{3}$ & EPM $^{4}$ & Valor de P \\
\hline AAC-glicose $(\mathrm{mg} / \mathrm{dL} / \mathrm{min})$ & 421,0 & 407,0 & 393,5 & 25,6 & 0,48 \\
AAC-insulina $(\mu \mathrm{UI} / \mathrm{dL} / \mathrm{min})$ & 152,8 & 141,3 & 156,5 & 54,0 & 0,88 \\
Triglicérides $(\mathrm{mg} / \mathrm{dL})$ & 33,4 & 48,5 & 47,8 & 15,5 & 0,08 \\
Colesterol total $(\mathrm{mg} / \mathrm{dL})$ & 99,7 & 95,5 & 89,6 & 10,5 & 0,27 \\
HDL $(\mathrm{mg} / \mathrm{dL})$ & 57,2 & 53,6 & 49,7 & 7,2 & 0,19 \\
VLDL $(\mathrm{mg} / \mathrm{dL})$ & 6,8 & 9,6 & 9,5 & 3,1 & 0,10 \\
LDL $(\mathrm{mg} / \mathrm{dL})$ & 35,8 & 32,3 & 30,5 & 9,5 & 0,58 \\
\hline
\end{tabular}

${ }^{1}$ Animais sem odontoplastia. ${ }^{2} 20$ dias após a odontoplastia. ${ }^{3} 40$ dias após a odontoplastia. ${ }^{4}$ Erro Padrão da Média. AAC: Área Abaixo da Curva. HDL: lipoproteína de alta densidade. VLDL: lipoproteína de muito baixa densidade. LDL: lipoproteína de baixa densidade.

Tabela 5 - Concentração de ácidos graxos de cadeia curta, ácido lático e $\mathrm{pH}$ nas fezes de cavalos submetidos ou não à odontoplastia (média $\pm \mathrm{EP}$ ).

\begin{tabular}{lcccc}
\hline \multicolumn{1}{c}{ Variável } & D0 $^{1}$ & $\mathbf{D 2 0}^{\mathbf{2}}$ & $\mathbf{D 4 0}^{3}$ & Valor de $\mathbf{P}$ \\
\hline Acetato (mmol) & $8,78 \pm 1,16$ & $8,70 \pm 1,42$ & $14,50 \pm 2,00$ & 0,07 \\
Propionato (mmol) & $3,37 \pm 0,29_{\mathrm{b}}$ & $3,26 \pm 0,36_{\mathrm{b}}$ & $5,06 \pm 0,51_{\mathrm{a}}$ & 0,03 \\
Butirato $(\mathrm{mmol})$ & $0,79 \pm 0,10$ & $0,63 \pm 0,10$ & $1,06 \pm 0,18$ & 0,19 \\
Lactato $(\mathrm{mmol})$ & $1,92 \pm 0,31_{\mathrm{a}}$ & $0,64 \pm 0,39_{\mathrm{b}}$ & $0,25 \pm 0,54_{\mathrm{b}}$ & 0,03 \\
$\mathrm{pH}^{4}$ & $6,58 \pm 0,14$ & $6,65 \pm 0,17$ & $6,34 \pm 0,12$ & 0,48 \\
\hline
\end{tabular}

${ }^{1}$ Animais sem odontoplastia. ${ }^{2} 20$ dias após a odontoplastia. ${ }^{3} 40$ dias após a odontoplastia. ${ }^{4}$ Média dos valores obtidos em 12 horas de avaliação. Médias seguidas por letras minúsculas na linha diferem entre si pelo teste de Tukey $(P<0,05)$.

\section{DISCUSSÃO}

No presente trabalho não foram identificados distúrbios comportamentais nos cavalos antes e após o procedimento dentário (tabela 2). $\mathrm{O}$ fato 
do consumo de água ter sido aumentado nos grupos tratados pode estar relacionado ao efeito diurético do cloridrato de Detomidina (alfa-2agonista), e que, segundo Marques et al. (2009), pode interferir tanto na hiperglicemia causando diurese osmótica, como na inibição do hormônio antidiurético, causando leve desidratação.

Os resultados obtidos para os CDA (tabela 3) corroboram os de Carmalt et al. (2004), onde não observaram diferenças nos CDA dos nutrientes sete e 19 semanas após o tratamento, e sugeriram que a odontoplastia não interfere na digestibilidade dos nutrientes da dieta de cavalos a curto prazo. Porém, Pagliosa et al. (2006) avaliaram 13 animais antes e após odontoplastia e encontraram aumento significativo no CDA dos nutrientes da dieta, com a utilização de indicador óxido crômico, e sugeriram que a melhora da trituração dos alimentos aumentou a exposição dos carboidratos estruturais aos processos digestivos, com melhor aproveitamento da forragem como fonte de energia.

Segundo Van Soest (1967) a fibra em detergente ácido (FDA) compreende a porção menos digestível da parede celular, sendo a celulose, lignina, cinzas, sílica e N-lignificado seus principais componentes. Segundo Drogoul et al. (2000; 2001) e Medina et al. (2002) fluidos e partículas finas fluem mais rapidamente do estômago para 0 duodeno, porém, apresentam maior tempo de retenção, especialmente no cólon. Assim, os resultados obtidos para CDA da FDA, concentração de propionato e acetato sugerem que a melhora na mastigação e trituração dos alimentos, quando os animais foram submetidos à odontoplastia, pode ter reduzido o tamanho das partículas de forma que passassem mais rapidamente pelo estômago, diminuindo os efeitos da acidez na parede celular da forragem, sem liberar totalmente os componentes indigestíveis. Porém, permaneceram por mais tempo no intestino grosso, favorecendo a fermentação dos carboidratos estruturais com aumento na concentração de acetato, observado no D40.

O propionato tem sua importância reconhecida devido ao fato de participar de rotas metabólicas, como a gliconeogênese. Evidências em pôneis indicam que em torno de $7 \%$ da glicose sanguínea é derivada do propionato. Além disso, a fermentação do amido não digerido produz lactato que pode ser absorvido juntamente com 0 produzido no local ou ser metabolizado a propionato pelas bactérias (Frape, 2008). A menor concentração de lactato observada nos cavalos submetidos à odontoplastia, sugere que não houve sobrecarga na digestão de amido no intestino delgado e, o lactato produzido, foi metabolizado a propionato, o que explicaria a maior concentração desse AGCC no D40. Porém, o aumento na concentração de propionato não afetou os valores de AAC de glicose e insulina.

O lactato, ao contrário dos ácidos acético, butírico ou propiônico, apresenta maior capacidade de causar alterações no $\mathrm{pH}$ do cólon devido ao seu baixo valor de $\mathrm{pKa}(3,86)$ tornandose, assim, um ácido mais forte do que os ácidos acético ( $\mathrm{pKa}=4,76)$, butírico (pKa=4,82) e propiônico (pKa=4,87) (Daly et al., 2012). Cavalos em pastejo apresentam pH intestinal entre 6,4 e 6,7. Valores inferiores a 6,0 foram associados com diarreia osmótica, supercrescimento de populações bacterianas indesejáveis e lise da população bacteriana desejável, aumentando o risco de endotoxemia e laminite (Hoffman, 2003; Julliand et al., 2006). A menor concentração de lactato nos cavalos submetidos à odontoplastia e o valor médio de $\mathrm{pH} \quad(6,52)$ encontrados neste estudo sugerem que não houve alterações gastrointestinais. 


\section{CONCLUSÃO}

Os padrões comportamentais e sanguíneos não foram alterados pela odontoplastia. As correções reduziram o coeficiente de digestibilidade aparente da FDA, a concentração de lactato fecal e aumentaram a concentração de propionato nas fezes, sugerindo melhor condições fermentativas no intestino grosso.

\section{REFERÊNCIAS}

ARAÚJO, F.C.D.; CRUZ, M.G.; BALIEIRO, J.C.C. et al. Effect of odontoplasty on apparent digestibility and consumption time of diet for equines. Arquivo Brasileiro de Medicina Veterinária e Zootecnia, v.70, n.1, p.29-36. 2018.

Association of Official Agricultural Chemists (AOAC): Official methods of analysis. 18ed. Gaithersburg, Maryland: AOAC International, 2005.

BENNET, D.G. Bits, Bridles and Accessories. In: BAKER, G.J AND EASLEY, J. Equine Dentistry. 2ed. Philadelphia: Elsevier, 2005, Cap. 2, p.9-22.

CARMALT, J.L.; TOWNSEND, H.G.; JANZEN, E.D. et al. Effect of dental floating on weight gain, body condition score, feed digestibility, and fecal particle size in pregnant mares. Journal of the American Veterinary Medical Association, v.225, n.12, p.1889-1893, 2004.

DALY, K.; PROUDMAN, C.J.; DUNCAN, S.H. et al. Alterations in microbiota and fermentation products in equine large intestine, in response to dietary variation and intestinal disease. British Journal of Nutrition, v.107, n.7, p.989-995, 2012.

DI FILIPPO, P.A.; VIEIRA, V.; RONDON, D.A. et al. Effect of dental correction on fecal fiber length in horses.
Journal of Equine Veterinary Science, v.64, p.77-80. 2018.

DIETRICH. L. O que é Odontoplastia? 2003. Disponível em: <http://www.cavalosdosul.com.br/artigo/ o-que-e-odontoplastia> Acesso em 04/04/2018.

DITTRICH, J.R.; MELO, H.A.; AFONSO, A.M.C.F. et al. Comportamento ingestivo de cavalos e a relação com 0 aproveitamento das forragens e bemestar dos animais. Revista Brasileira de Zootecnia, v.39, p.130-137, 2010 (Suplemento Especial).

DROGOUL, C.; DE FOMBELLE, A.; JULLIAND, $V$. Feeding and microbial disorders in horses: 2. Effect of three hay:grain ratios on digesta passage rate and digestibility in ponies. Journal of Equine Veterinary Science, v.21, n.10, p.487-491, 2001.

DROGOUL, C.; PONCET, C.; TISSERAND, J.L. Feeding ground and pelleted hay rather than chopped hay to ponies 1. Consequences for in vivo digestibility and rate of passage of digesta. Animal Feed Science and Technology, v.87, n. 1-2, p.117-130, 2000.

ERWIN, E.S.; MARCO, G.J.; EMERY, E.M. Volatile fatty acid analyses of blood and rumen fluid by gas chromatography. Journal of Dairy Science, v.44, n.9, p.1768-1771, 1961.

FRAPE D. Nutrição \& alimentação de cavalos. 3ed. São Paulo: Roca, 2008. $602 p$.

GETACHEW, G.; MAKKAR, H.P.S.; BECKER, K. Tropical browses: contents of phenolic compounds, in vitro gas production and stoichiometric relationship between short chain fatty acid and in vitro gas production. Journal of Agricultural Science, v.139, p.341352, 2002.

HOFFMAN, R.M. Carbohydrate metabolism in horses. In: TALSTON, 
S.L., HINTZ, H.F. Recent advances in equine nutrition. Ithaca: International Veterinary Information Service, 2003.

HYDOCK, K.L.; NISSLEY, S.G.; STANIAR, W.B. A standard protocol for fecal $\mathrm{pH}$ measurement in the horse. The Professional Animal Scientist, v.30, p.643-648, 2014.

JULLIAND, V.; DE FOMBELLE, A.; VARLOUD, $M$. Starch digestion in horses: The impact of feed processing. Livestock Science, v.100, p.44-52, 2006.

MARCHELLO, E.V.; SCHURG, W.A.; MARCHELLO, I.A. et al. Changes in lipoprotein composition in horses fed a fat-supplemented diet. Journal of Equine Veterinary Science, v. 20, n.7, p.453-458, 2000.

MARQUES, J.A.; PEREIRA, D.A.; MARQUES, I.C.S. Associação entre midazolam e detomidina na medicação pré-anestésica para indução da anestesia geral com cetamina em potros. Arquivo Brasileiro de Medicina Veterinária e Zootecnia, v.61, n.6, p.1290-1296, 2009.

MATTHEWS, J.N.S; ALTMAN, D.G.; CAMPBELL, M.J. et al. Analysis of serial measurements in medical research. British Medical Journal, v.300, p.230235, 1990.

MEDINA, B.; GIRARD, I.D.; JACOTOT, $E$. et al. Effect of a preparation of Saccharomyces cerevisiae on microbial profiles and fermentation patterns in the large intestine of horses fed a high fiber or a high starch diet. Journal of Animal Science, v.80, n.10, p.2600-2609. 2002.

National Research Council (NRC). Nutrient Requirements of Horses. 6ed, Washington, D.C.: National Academies Press, 2007.

PAGLIOSA, G.M.; ALVES, G.E.S.; FALEIROS, R.R. et al. Influência das pontas excessivas de esmalte dentário na digestibilidade e nutrientes de dietas de cavalos. Arquivo Brasileiro de Medicina Veterinária e Zootecnia, v.58, n.1, p.94-98, 2006.

PRYCE, J.D. A modification of the Barker-Summerson method for the determination of lactic acid. Analyst, v.94, n.1125, p.1151-1152, dezembro, 1969.

VAN SOEST, P.J. Development of a comprehensive system of feed analysis and its application to forages. Journal of Animal Science, v.26, p.119-128, 1967.

VAN SOEST, P.J.; ROBERTSON, J.B.; LEWIS, B.A. Methods for dietary fiber, and nonstarch polysaccharides in relation to animal nutrition. Journal of Dairy Science, v.74, n.10, p.3583-3597, 1991. 